\title{
Analysis of Non-One-Dimensional Shear Concentration Convective Flows of a Viscous Incompressible Fluid in a Plane Horizontal Layer with Motionless Boundaries
}

\author{
N. V. Burmasheva, ${ }^{1,2)}$ and E. Yu. Prosviryakov ${ }^{1,2, b)}$ \\ ${ }^{1}$ Institute of Engineering Science, Ural Branch of the Russian Academy of Sciences, \\ 34 Komsomolskaya St., Ekaterinburg, 620049, Russian Federation \\ ${ }^{2}$ Ural Federal University named after the first President of Russia B.N.Yeltsin, \\ 19 Mira St., Ekaterinburg, 620002, Russian Federation

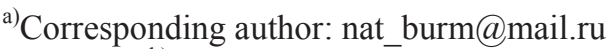 \\ b)evgen_pros@mail.ru
}

\begin{abstract}
Increased mineralization of some fluid layers in comparison with that of the other layers causes the appearance of concentration-induced convection. This type of convection is most pronounced for brines of surface water bodies, salt marshes, saline rocks, etc. Nevertheless, concentration-induced convection occurs not only in natural reservoirs, but also in technical devices due to the inhomogeneous distribution of various impurities in hydraulic media. The characteristics of impurity distribution have a significant effect on the flow characteristics. The paper considers a shear convective flow of a viscous incompressible fluid in a horizontal layer, which is induced by the nonuniform distribution of impurity concentration. A system of equations for concentration-induced convection is used to describe such flows; a solution is sought among classes of generalized solutions. As the boundary conditions, it is assumed that the no-slip condition is satisfied at the lower impermeable boundary, that the upper boundary of the layer is motionless, and that the distribution of salinity and pressure is specified on it. A solution to the described boundary value problem is obtained. The attention is focused on the analysis of the properties of the flow velocity field. The conditions at which the flow reduces to a unidirectional one are derived. It is shown that none of the velocity field components can vanish inside the fluid layer, i.e. that the fluid layer cannot be divided into zones in such a way that the fluid would change the flow direction when transiting from one zone to another.
\end{abstract}

\section{BOUNDARY VALUE PROBLEM FORMULATION}

The steady-state shear diffusion flow of a viscous incompressible fluid in a plane horizontal layer of thickness $h$ is considered. The model is based on using the system of Navier-Stokes equations in the Boussinesq approximation, the incompressibility equation, and the equation for the change in the concentration of the light phase of a binary mixture [1],

$$
\begin{gathered}
(\boldsymbol{V} \cdot \nabla) \boldsymbol{V}=-\nabla P+v \Delta \boldsymbol{V}+g \beta C \boldsymbol{k} ; \\
(\boldsymbol{V} \cdot \nabla) C=d \Delta C ; \\
\nabla \cdot \boldsymbol{V}=0 .
\end{gathered}
$$

Here, $\boldsymbol{V}=\left(V_{x}(x, y, z), V_{y}(x, y, z), 0\right)$ is the velocity vector; $P(x, y, z)$ is the deviation of pressure from hydrostatic, divided by the average density; $C(x, y, z)$ is the concentration of the light phase of a binary mixture; $v, d$ are the kinematic (molecular) viscosity of the fluid and the diffusion coefficient, respectively; $g$ is the acceleration of gravity; $\nabla, \Delta$ are the Hamilton and Laplace three-dimensional operators, respectively.

The solution is sought in the class [2-4] 


$$
\begin{gathered}
V_{x}=U(z) ; V_{y}=V(z) ; V_{z}=0 ; \\
P=P_{0}(z)+x P_{1}(z)+y P_{2}(z) ; C=C_{0}(z)+x C_{1}(z)+y C_{2}(z) .
\end{gathered}
$$

Note that the nonlinear system of partial differential equations (1) includes five scalar equations for four unknown functions, namely velocities $V_{x}$ and $V_{y}$, concentration $C$, and pressure $P$. This means that system(1) is overdetermined. However, for the velocity class of the form represented by Eq. (2), the incompressibility equation is satisfied identically; thus, the balance between the number of equations and the number of unknowns for class (2) is satisfied.

It can be rigorously shown that, by substituting the selected representation of hydrodynamic fields (2)-(3) into system (1), we can reduce this system to the following equivalent system of ordinary differential equations for the components of expansion (2)-(3):

$$
\begin{gathered}
C_{1}{ }^{\prime}=0 ; C_{2}{ }^{\prime \prime}=0 ; P_{1}{ }^{\prime}=g \beta C_{1} ; P_{2}{ }^{\prime}=g \beta C_{2} ; \\
v V^{\prime \prime}=P_{2}, v U^{\prime \prime}=P_{1}, d C_{0}{ }^{\prime \prime}=U C_{1}+V C_{2}, P_{0}{ }^{\prime}=g \beta C_{0} .
\end{gathered}
$$

The prime in system (4) denotes differentiation with respect to the vertical (transverse) coordinate $z$. The equations of system (4) are integrated according to the given order.

In order to obtain a solution to system (4) adequate to the actual diffusion flow, it is necessary to add the corresponding number of boundary conditions. Let the steady diffusion flow of a viscous binary fluid in an infinite horizontal layer be further considered as an example.

Assume that the solid boundary $z=0$ of the fluid layer is impermeable and that the fluid no-slip condition is fulfilled on it [5-7],

$$
\left.V_{x}\right|_{z=0}=\left.V_{y}\right|_{z=0}=0,\left.\frac{\partial C}{\partial \boldsymbol{n}}\right|_{z=0}=0 .
$$

Here, $\boldsymbol{n}$ is the normal to the lower boundary of the layer.

Assume that a uniform pressure corresponding to atmospheric pressure and an inhomogeneous distribution of impurity concentration are set at the upper immobile boundary $z=h$ as

$$
\left.V_{x}\right|_{z=h}=\left.V_{y}\right|_{z=h}=0,\left.P\right|_{z=h}=S_{0},\left.C\right|_{z=h}=a x+b y .
$$

\section{EQUATION SYSTEM SOLUTION}

The solution of the boundary value problem (4)-(6) takes the following form:

$$
\begin{gathered}
U=\psi Z(-2+Z)(-1+Z) ; \\
V=\gamma Z(-2+Z)(-1+Z) ; \\
C_{1}=a ; C_{2}=b ; \\
C_{0}=\lambda(-1+Z)\left(8+8 Z+8 Z^{2}-12 Z^{3}+3 Z^{4}\right) ; \\
P_{1}=\xi_{1}(-1+Z) ; P_{2}=\xi_{2}(-1+Z) ; \\
P_{0}=S_{0}+\xi_{3}(-1+Z)^{2}\left(11+6 Z+Z^{2}-4 Z^{3}+Z^{4}\right) .
\end{gathered}
$$

Here, 


$$
\begin{gathered}
Z=z / h, \psi=a g \beta h^{3} /(6 v), \gamma=b g \beta h^{3} /(6 v), \lambda=\left(a^{2}+b^{2}\right) g h^{5} \beta /(360 d v), \\
\xi_{1}=a g \beta h, \xi_{2}=b g \beta h, \xi_{3}=\left(a^{2}+b^{2}\right) g^{2} h^{6} \beta^{2} /(720 d v) .
\end{gathered}
$$

In what follows, a non-one-dimensional field of flow velocities is analyzed in detail.

\section{INVESTIGATION OF THE VELOCITY FIELD}

Note that, with an accuracy of up to a constant factor, the velocities $U$ and $V$ are described by one and the same polynomial. Let it be denoted as

$$
f(Z)=Z(-2+Z)(-1+Z) \text {. }
$$

The coefficients $\psi$ and $\gamma$ in solution (7) for the velocities $U$ and $V$ describe the influence of the impurity distribution law at the upper boundary on the flow properties. If there is such a nonzero number $k$ that $\psi=k \gamma$, the flow under study is easily reducible to a unidirectional one by transforming the rotation of the horizontal axes.

The behavior of the profile of the velocity field components is determined by the properties of the polynomial $f$. It is obvious that, at the boundaries of the layer (at $Z=0$ and $Z=1$ ), it vanishes. The remaining root of this polynomial falls beyond the studied interval $(0,1)$. Thus, the velocities $U$ and $V$ do not vanish anywhere except at the layer boundaries. This means that everywhere in the layer each of the velocities can take values of only one sign (Fig. 1).

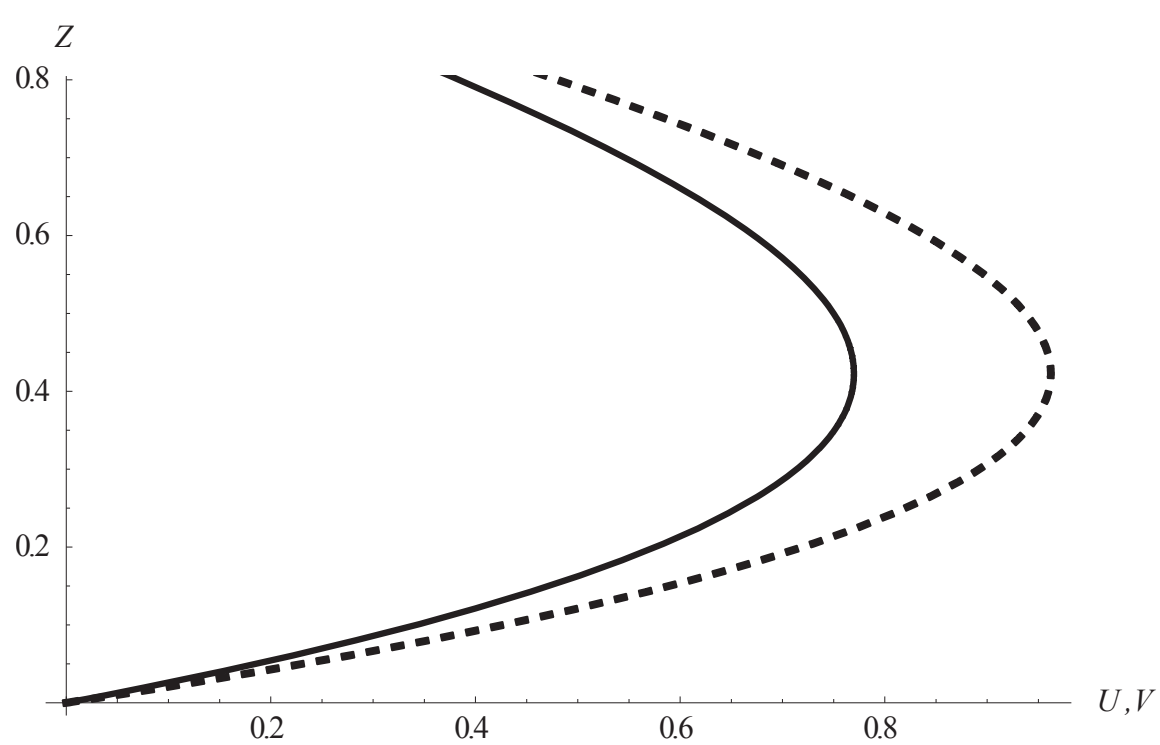

FIGURE 1. The behavior of the velocities $U$ (solid line) and $V$ (dashed line)

The distribution of streamlines (Fig. 2) illustrates the fact that the exact solution (7) for the velocity field (2) does not allow stratification [8-12] at any values of the concentration field. 


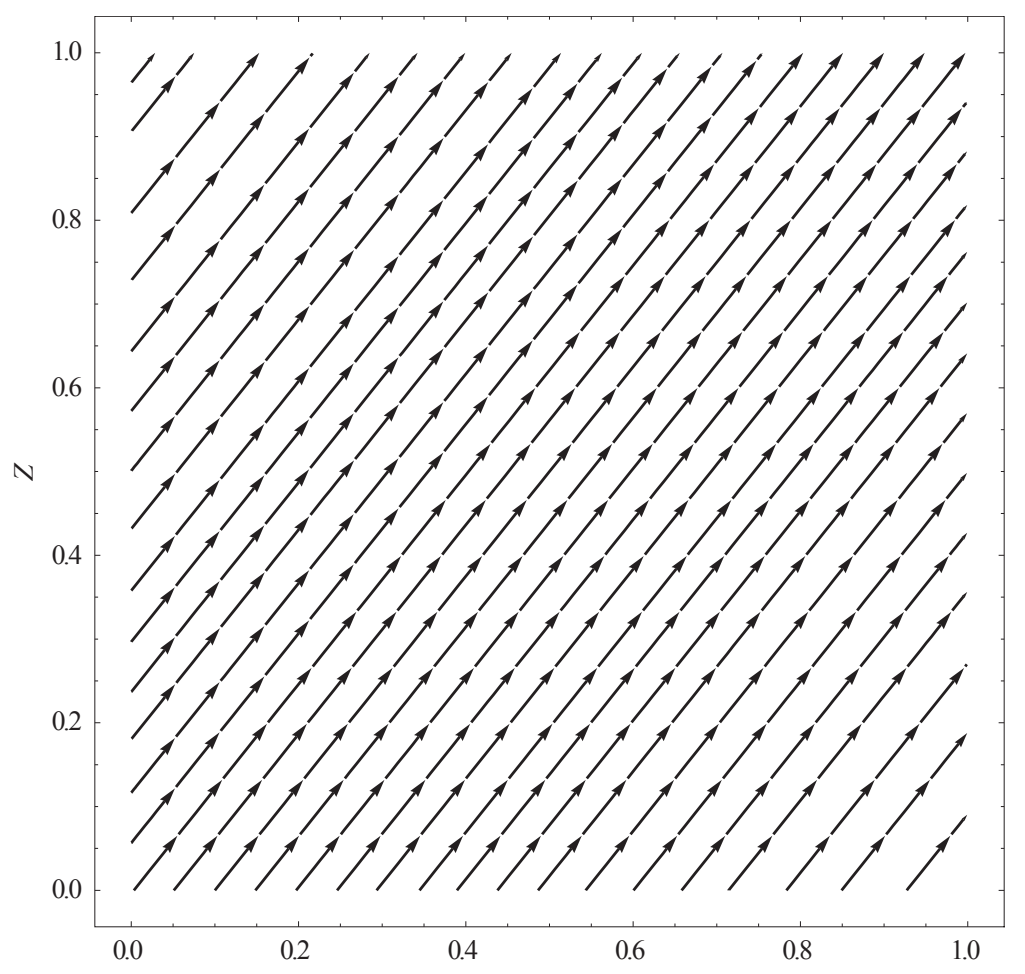

FIGURE 2. Streamlines of the flow velocity field

Note that, despite the absence of stratification points, the specific kinetic energy $E_{\mathrm{k}}$ has a nonmonotonic behavior (Fig. 3) and, at the same time, takes zero values only at the boundaries of the fluid layer under study.

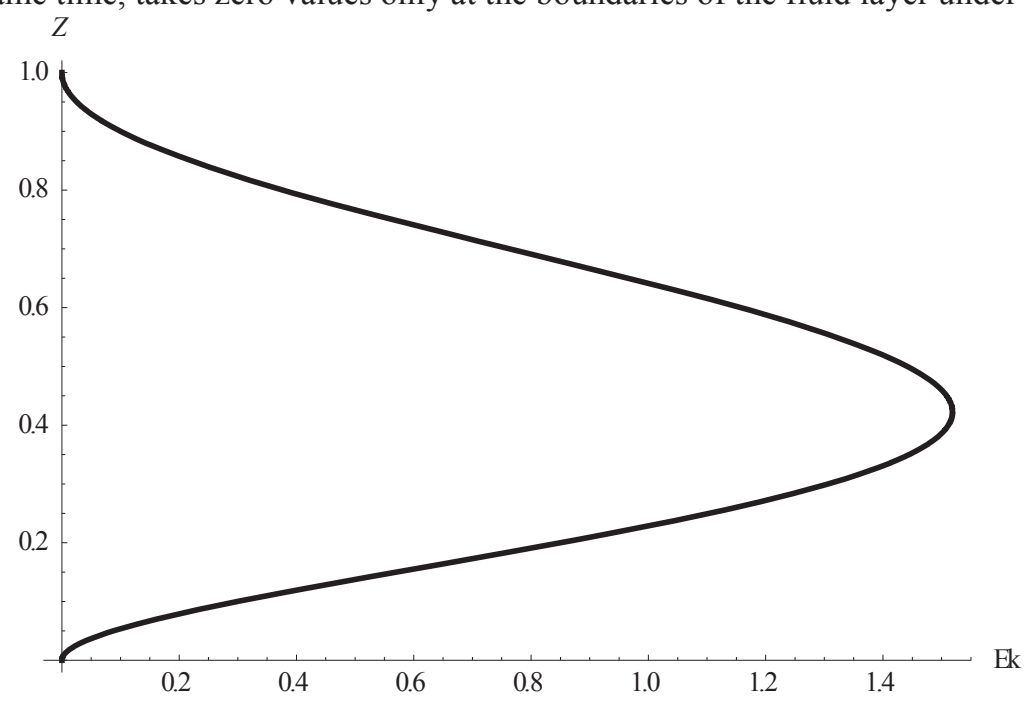

FIGURE 3. Kinetic energy profile

CONCLUSION

The paper has presented an exact solution for steady-state diffusion flows of a viscous incompressible fluid. It has been shown that there are no such values of concentration that this solution would allow the appearance of reverse flow regions. 


\section{REFERENCES}

1. G. Z. Gershuni and E. M. Zhukhovitskii, Convective Stability of Incompressible Fluids (Wiley/Keter Press, Jerusalem, 1976).

2. N. V. Burmasheva and E. Yu. Prosviryakov, Vestn. Samar. Gos. Tekhn. Univ., Ser. Fiz.-Mat. Nauki 21(1), 180-196 (2017).

3. N. V. Burmasheva and E. Yu. Prosviryakov, Vestn. Samar. Gos. Tekhn. Univ., Ser. Fiz.-Mat. Nauki 21(4), 736-751 (2017).

4. N. V. Burmasheva, E. A. Larina, and E. Yu. Prosviryakov, AIP Conference Proceedings 2176, 030023 (2019).

5. S. V. Goldstein, Modern Developments in Fluid Mechanics (Oxford Univ. Press, Oxford, 1938).

6. L. Prandtl and O. Tietjens, Fundamentals of Hydro- and Aeromechanics (McGraw-Hill Book Company, New York - London, 1934).

7. E. I. Borzenko and G. R. Shrager, Computational Continuum Mechanics 12(2), 129-136 (2019).

8. G. A. Ostroumov, Free convection under the condition of the internal problem (National Advisory Committee for Aeronautics, Washington, NACA Technical Memorandum 1407, 1958).

9. R. V. Birikh, J. Appl. Mech. Tech. Phys. 7(3), 43-44 (1966).

10. V. Shtern, Counterflows. Paradoxical Fluid Mechanics Phenomena (Cambridge University Press, Cambridge 2012).

11. J. M. Dorrepaal, Journal of Fluid Mechanics 163(1), 141-147 (1986).

12. J. T. Stuart, Journal of the Aerospace Sciences 26(2), 124-125 (1959). 